\title{
Long-term effect of CFTR modulator therapy on airway nitric oxide
}

To the Editor:

The fraction of exhaled nitric oxide $\left(F_{\mathrm{eNO}}\right)$ is generally lower in individuals with cystic fibrosis $(\mathrm{CF})$, compared to healthy controls. Two recent studies reported that the cystic fibrosis transmembrane conductance regulator (CFTR) potentiator ivacaftor resulted in an increase in $F_{\text {eNO }}$ after 4 weeks' therapy $[1,2]$, suggesting that changes in $F_{\mathrm{eNO}}$ have the potential to serve as biomarker of restored CFTR function. However, it is currently unknown whether ivacaftor results in a sustained increase in $F_{\mathrm{eNO}}$ and whether combination therapy of ivacaftor with the CFTR corrector lumacaftor also leads to changes in $F_{\mathrm{eNO}}$. Therefore, the objective of this research was to document long-term effects of ivacaftor and lumacaftorivacaftor therapy on $F_{\mathrm{eNO}}$ in treated CF patients. The two prospective observational studies were approved by the local institutional review boards (Hospital for Sick Children's research ethics board \#1000036224 and \#1000057599, St Michael's Hospital research ethics board \#13-089). Patients were included if they had a confirmed diagnosis of $\mathrm{CF}$ and were eligible for treatment with either therapy. $F_{\mathrm{eNO}}$ was measured before and 1, 3, 6, 12 and 24 months after initiation of therapy during regular outpatient visits. Sputum samples were collected in the ivacaftor cohort. The nonliquid phase (mucus plugs) of the sputum was processed by adding $0.1 \%$ dithiothreitol in Dulbecco's PBS (4:1, vol:wt), and the clear supernatant of the cell suspension was separated from the cells by centrifugation $[3,4]$. No protease inhibitors were added. Samples were stored at $-80^{\circ} \mathrm{C}$ before analysis of $\mathrm{L}$-arginine metabolism using liquid chromatography-mass spectrometry (LC-MS), as reported [5]. Nitric oxide (NO) metabolites nitrate and nitrite were measured in sputum using Griess reagent [6] and myeloperoxidase (MPO) was measured using ELISA (R\&D Systems, Minneapolis, MN, USA). Changes in outcomes between visits were assessed using paired t-tests and Wilcoxon signed-rank tests for skewed distributions, and correlations were assessed using Pearson's correlation coefficient.

34 patients treated with CFTR modulator therapy were included. The ivacaftor cohort consisted of eight paediatric (median (interquartile range (IQR)) age 12.5 (9.4-14.0) years, 63\% female) and 12 adult (age 32.6 (24.6-42.8) years, $58 \%$ female) patients. In the lumacaftor-ivacaftor cohort there were 14 paediatric patients (age 14.5 (14.0-16.9) years, 57\% female) and no adults.

At 1 month follow-up the ivacaftor cohort consisted of seven children and nine adult patients; at 1 year six children and five adults; and at 2 years six children and four adults. Forced expiratory volume in $1 \mathrm{~s}$ $\left(\mathrm{FEV}_{1}\right)$ at baseline was $78(71-90) \%$ predicted in the paediatric patients and $62(47-74) \%$ pred in the adult patients. $\mathrm{FEV}_{1}$ was improved for the total group at 1 month, from $69.7 \%$ pred to $81.1 \%$ pred (change 11.4 , 95\% CI 6.1-16.8; $\mathrm{p}<0.001$ ), and remained improved for the entire follow-up. Similar results were seen for forced vital capacity (FVC), with an improvement from $88.9 \%$ pred to $97.8 \%$ pred (change $8.9,95 \%$ CI 5.0-12.8; $\mathrm{p}<0.001)$ at 1 month, which persisted throughout follow-up.

$F_{\text {eNO }}$ was increased compared to baseline after 4 weeks on ivacaftor $(n=16)$, as previously reported [1], and remained increased throughout follow-up (figure 1 ). When including all measurements, $F_{\mathrm{eNO}}$ weakly correlated with FVC $(\mathrm{r}=0.29, \mathrm{p}=0.009)$ and $\mathrm{FEV}_{1}(\mathrm{r}=0.41, \mathrm{p}<0.001 ; \mathrm{n}=81)$, but changes in $F_{\mathrm{eNO}}$ did not correlate with changes in pulmonary function tests over time $\left(r=0.19, p=0.13\right.$ for $F_{E V} ; r=0.14, p=0.28$ for FVC \% pred; $\mathrm{n}=64)$.

A mild decrease at 1 month was seen in sputum L-arginine, a substrate for NO synthases (NOS) (baseline $14.7(8.1-40.7) \mu \mathrm{mol} \cdot \mathrm{L}^{-1}$, median change $\left.-5.4, \mathrm{p}=0.04 ; \mathrm{n}=13\right)$, and an insignificant decrease for the NOS

@ERSpublications

Ivacaftor results in a sustained increase in $F_{\mathrm{eNO}}$ in children and adults with CF. The increase in $F_{\mathrm{eNO}}$ may be related to changes in airway NO metabolism by myeloperoxidase. Lumacaftor-ivacaftor therapy does not have an immediate effect on $F_{\text {eNO }}$. http://bit.ly/2mzqhds

Cite this article as: Grasemann $\mathrm{H}$, Klingel M, Avolio J, et al. Long-term effect of CFTR modulator therapy on airway nitric oxide. Eur Respir J 2020; 55: 1901113 [https://doi.org/10.1183/13993003.01113-2019]. 


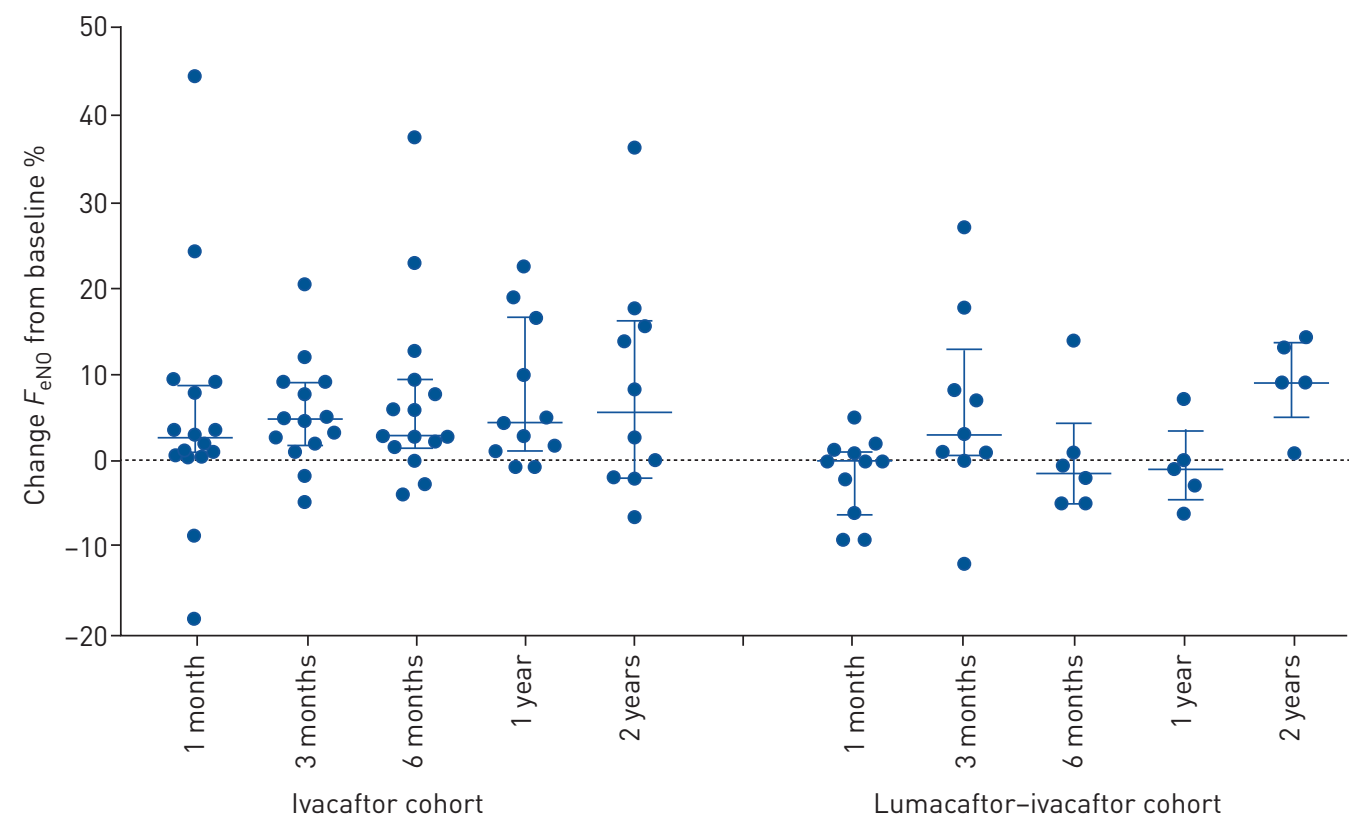

FIGURE 1 Changes in the exhaled nitric oxide fraction $\left(F_{\mathrm{eNO}}\right)$ in patients with cystic fibrosis treated with ivacaftor or lumacaftor-ivacaftor. Each data point represents an individual measurement. Also shown are median and interquartile range changes from before treatment baseline. Median $F_{\mathrm{eNo}}$ was increased from baseline at all time points in ivacaftor-treated patients. A significant increase in $F_{\mathrm{eNO}}$ in the lumacaftorivacaftor-treated patients was seen only at 2 years follow-up.

inhibitor asymmetry dimethylarginine (ADMA) (baseline $0.08(0.01-0.26) \mu \mathrm{mol} \cdot \mathrm{L}^{-1}$, median change $-0.01 ; \mathrm{p}=0.06$ ). While there was a correlation between $F_{\mathrm{eNO}}$ and sputum L-arginine/ADMA ratio, an index of NOS impairment $(\mathrm{r}=0.31, \mathrm{p}=0.03 ; \mathrm{n}=51)$, which was supportive of previous observations [5], L-arginine/ ADMA remained unchanged during follow-up.

MPO was decreased at 1 month (baseline $27.0(10.7-52.2) \mu \mathrm{g} \cdot \mathrm{mL}^{-1}$, median change $-4.8 ; \mathrm{p}=0.03$ ) in the total group $(n=9)$, but at no other time point. There was no change in sputum levels of nitrite or nitrate in the total group, but reduced nitrite at 4 weeks in adults (baseline $22.8(21.0-37.2) \mu \mathrm{mol} \cdot \mathrm{L}^{-1}$, median change -9.1, $\mathrm{p}=0.03)$. Nitrite/nitrate ratio was decreased from baseline at 4 weeks $(\mathrm{p}=0.06)$, but at no other visit and in adults only $(\mathrm{n}=7)$. There was a significant reverse correlation between sputum MPO and $F_{\text {eNO }}(\mathrm{r}=-0.34, \mathrm{p}=0.02 ; \mathrm{n}=45)$, suggesting that a decrease in MPO content may contribute to the increase in $F_{\mathrm{eNO}}$ with ivacaftor therapy.

Median (IQR) $\mathrm{FEV}_{1}$ for the lumacaftor-ivacaftor cohort was 80.1 (68.6-93.8)\% pred at baseline and was not significantly different at any time point after initiation of therapy. There was no change in FVC \% pred during follow-up.

Median (IQR) $F_{\text {eNO }}$ before lumacaftor-ivacaftor $(10(8-15) \mathrm{ppb})$ was similar to ivacaftor baseline values, but did not change within the first year of treatment (figure 1). However, increased $F_{\mathrm{eNO}}$ from baseline was found for five patients at the 2-year follow-up (median increase $9 \mathrm{ppb}, 95 \%$ CI 2.8-15.6 ppb; $\mathrm{p}=0.02$ ). Changes in $F_{\mathrm{eNO}}$ did not correlate with changes in $\mathrm{FEV}_{1}(\mathrm{r}=0.14, \mathrm{p}=0.35)$.

Alterations in L-arginine/NO metabolism and reduced $\mathrm{NO}$ availability are thought to be clinically important in CF, as low NO may contribute to airways obstruction and favour colonisation with certain pathogens. While the exact reasons for the decreased $F_{\text {eNO }}$ in CF are currently not known, studies have shown that 4 weeks of therapy with the CFTR potentiator ivacaftor resulted in an increase of $F_{\mathrm{eNO}}[1,2,7]$. We here present data showing that the increase in $F_{\mathrm{eNO}}$ on ivacaftor therapy is not short-lived or transient, but rather sustained over a 2-year follow-up. Furthermore, using LC-MS analyses in sputum we demonstrate that the long-term increase in $F_{\mathrm{eNO}}$ is not due to changes in L-arginine availability or L-arginine metabolism in the airways. However, our data suggest that changes in airway metabolism of NO by MPO may be a cause of the observed increase in $F_{\mathrm{eNO}}$.

Previous studies have shown that the relative quantities of $\mathrm{NO}, \mathrm{NO}_{2}{ }^{-}$and $\mathrm{NO}_{3}{ }^{-}$provide mechanistic insight into $\mathrm{NO}$ metabolism in the $\mathrm{CF}$ lung and that $\mathrm{NO}$ catabolism within the airway prior to exhalation may contribute to reduced $F_{\mathrm{eNO}}$ in CF. NO oxidised by MPO results in $\mathrm{NO}_{2}{ }^{-}$and continued oxidation by 
MPO and other oxidative processes results in $\mathrm{NO}_{3}^{-}$formation $[8,9]$. We observed a decrease in sputum MPO levels, decreased nitrite and thus relatively more nitrate (no change in total $\mathrm{NO}_{2}{ }^{-}+\mathrm{NO}_{3}{ }^{-}$), which suggests that liberation of $\mathrm{NO}$ from $\mathrm{NO}_{2}{ }^{-}$stored in airway secretions may contribute to the observed increase in $F_{\mathrm{eNO}}$ at 4 weeks of therapy. Although changes in MPO were not significant at other time points, a potential role of MPO for the observed changes in NO metabolism was supported by a reverse correlation of MPO sputum levels and $F_{\mathrm{eNO}}$. However, our observations are based on relatively low numbers and do not exclude the possibility that other mechanisms such as changes in airway $\mathrm{pH}$, inflammation and increased NO synthase expression or function may contribute to the long-term effect of ivacaftor therapy on airway NO. In addition, the decreasing number of CF adults during follow-up may introduce a bias towards positive changes in $F_{\mathrm{eNO}}$ over time, as the effect of ivacaftor on airway NO may be more pronounced in children compared to adults with CF [1].

In contrast to ivacaftor, lumacaftor-ivacaftor therapy did not result in a consistent change in $F_{\mathrm{eNO}}$. This may be related to differences in efficacy, as lumacaftor-ivacaftor it is known to have less significant effects on respiratory outcomes in patients homozygous for F508del, compared to ivacaftor monotherapy in patients with gating mutations [10]. The lack of effect on $F_{\mathrm{eNO}}$ in children within the first year of treatment was consistent with a recent observation in four adults with $\mathrm{CF}$ [7]. However, $F_{\mathrm{eNO}}$ was significantly increased from baseline in a small number of our paediatric patients at 2 years follow-up $(\mathrm{n}=5)$. Whether longer term treatment with lumacaftor-ivacaftor results in a sustained effect on $F_{\mathrm{eNO}}$ will need to be shown in future studies.

Ivacaftor results in a sustained increase in $F_{\mathrm{eNO}}$ in children and adults with CF. The increase in $F_{\mathrm{eNO}}$ with ivacaftor may be related to changes in airway NO metabolism by MPO. Lumacaftor-ivacaftor did not have an immediate effect on $F_{\mathrm{eNO}}$ in treated children, supporting findings in adults with $\mathrm{CF}$.

\footnotetext{
Hartmut Grasemann ${ }^{1,2}$, Michelle Klingel ${ }^{2}$, Julie Avolio ${ }^{2}$, Carley Prentice ${ }^{1}$, Tanja Gonska ${ }^{2,3}$, Elizabeth Tullis ${ }^{4}$ and Felix Ratjen ${ }^{1,2}$

${ }^{1}$ Division of Respiratory Medicine, Dept of Pediatrics, The Hospital for Sick Children, University of Toronto, Toronto, ON, Canada. ${ }^{2}$ Translational Medicine, SickKids Research Institute, The Hospital for Sick Children, University of Toronto, Toronto, ON, Canada. ${ }^{3}$ Division of Gastroenterology, Dept of Pediatrics, The Hospital for Sick Children, University of Toronto, Toronto, ON, Canada. ${ }^{4}$ Division of Respirology and Keenan Research Centre of Li Ka Shing Knowledge Institute, Dept of Medicine, St Michael's Hospital, University of Toronto, Toronto, ON, Canada.
}

Correspondence: Hartmut Grasemann, Hospital for Sick Children, 555 University Avenue, Toronto, ON M5G 1X8, Canada. E-mail: hartmut.grasemann@sickkids.ca

Received: 11 June 2019 | Accepted after revision: 18 Sept 2019

Acknowledgements: We thank all patients and families who participated in this research study. Mass spectrometry analyses were performed at the Analytical Facility for Bioactive Molecules (AFBM) of the Centre for the Study of Complex Childhood Diseases (CSCCD) at the Hospital for Sick Children (Toronto, ON, Canada). The CSCCD was supported by the Canadian Foundation for Innovation (CFI)

Support statement: Parts of this study were supported by Vertex Pharmaceuticals (Vertex Investigator Initiated Studies Program). Funding information for this article has been deposited with the Crossref Funder Registry.

Conflict of interest: H. Grasemann reports grants from Vertex Pharmaceuticals, during the conduct of the study. M. Klingel has nothing to disclose. J. Avolio has nothing to disclose. C. Prentice has nothing to disclose. T. Gonska received funding from Vertex Pharmaceuticals, for parts of the ivacaftor observational study. E. Tullis reports grants and personal fees from Vertex Pharmaceuticals, during the conduct of the study; grants and personal fees from Proteostasis, outside the submitted work. F. Ratjen reports grants and personal fees for consultancy from Vertex, personal fees for consultancy from Novartis, Bayer, Roche and Genetech, outside the submitted work.

\section{References}

1 Grasemann $\mathrm{H}$, Gonska T, Avolio J, et al. Effect of ivacaftor therapy on exhaled nitric oxide in patients with cystic fibrosis. J Cyst Fibros 2015; 14: 727-732.

2 Kotha K, Szczesniak RD, Naren AP, et al. Concentration of fractional excretion of nitric oxide $\left(F_{\mathrm{eNO}}\right)$ : a potential airway biomarker of restored CFTR function. J Cyst Fibros 2015; 14: 733-740.

3 Pizzichini E, Pizzichini MM, Efthimiadis A, et al. Indices of airway inflammation in induced sputum: reproducibility and validity of cell and fluid-phase measurements. Am J Respir Crit Care Med 1996; 154: 308-317.

4 Hilliard JB, Konstan MW, Davis PB. Inflammatory mediators in CF patients. Methods Mol Med 2002; 70: 409-431.

5 Grasemann $\mathrm{H}$, Al-Saleh S, Scott JA, et al. Asymmetric dimethylarginine contributes to airway nitric oxide deficiency in patients with cystic fibrosis. Am J Respir Crit Care Med 2011; 183: 1363-1368.

6 Grasemann C, Ratjen F, Schnabel D, et al. Effect of growth hormone therapy on nitric oxide formation in cystic fibrosis patients. Eur Respir J 2008; 31: 815-821.

7 Vincken S, Verbanck S, De Wachter E, et al. Exhaled nitric oxide in stable adult cystic fibrosis patients, during exacerbation and following CFTR-modifying treatment. Eur Respir J 2019; 53: 1802259. 
Chapman AL, Morrissey BM, Vasu VT, et al. Myeloperoxidase-dependent oxidative metabolism of nitric oxide in the cystic fibrosis airway. J Cyst Fibros 2010; 9: 84-92.

9 Cantin AM, White TB, Cross CE, et al. Antioxidants in cystic fibrosis. Conclusions from the CF antioxidant workshop, Bethesda, Maryland, November 11-12, 2003. Free Radic Biol Med 2007; 42: 15-31.

10 Wainwright CE, Elborn JS, Ramsey BW, et al. Lumacaftor-ivacaftor in patients with cystic fibrosis homozygous for Phe508del CFTR. N Engl J Med 2015; 373: 220-231.

Copyright $@$ ERS 2020 